\title{
Hemoglobin Affinity for Oxygen in the Anginal Syndrome with Normal Coronary Arteriograms
}

\author{
Pantel S. Vokonas, Peter F. Cohn, Michael D. Klein, \\ Myron B. LAVER, and Ruchard GoruIN \\ From the Cardiovascular Division, Department of Medicine, Peter Bent Brigham \\ Hospital, and the Anesthesia Laboratory, Department of Anesthesia, \\ Massachusetts General Hospital, and Harvard Medical School, \\ Boston, Massachusetts 02115
}

\begin{abstract}
A в S T R A c T Oxyhemoglobin dissociation (OHD) curves were performed on whole blood (WB) from 20 patients with anginal pain, normal hemodynamics, and normal coronary arteries, as demonstrated by selective coronary cinearteriography. OHD curves in 19 of 20 patients, from zero to full saturation, were nearly identical to those in normal control subjects with values for $\mathrm{P}_{80}\left(\mathrm{PO}_{0}\right.$ at $50 \%$ saturation and $\mathrm{pH} 7.4$ ) of $26.7 \pm$ 1.5 (mean $\pm \mathrm{SD}$ of the mean) torr $(\mathrm{mm} \mathrm{Hg}$ ) and red blood cell (RBC) levels of 2,3-diphosphoglyceric acid (2, 3-DPG) of $0.72 \pm 0.10$ (mean $\pm \mathrm{SD}$ of the mean) $\mathrm{M} / \mathrm{M}$ hemoglobin $(\mathrm{Hb})$. Normal values for nonsmoking adults were: $P_{50}, 26.6 \pm 1.4$ (mean $\pm S D$ of the mean) torr; and RBC 2,3-DPG, $0.81 \pm 0.09$ (mean $\pm \mathrm{SD}$ of the mean) $\mathrm{M} / \mathrm{M} \mathrm{Hb}$. Mean levels of carbon monoxide were normal at $0.14 \pm 0.01$ (mean \pm SEM) $\mathrm{ml} / 100 \mathrm{ml} \mathrm{WB}$ in 10 patients who were nonsmokers and $0.45 \pm 0.15$ (mean \pm SEM) $\mathrm{ml} / 100 \mathrm{ml} \mathrm{WB}$ in 10 smokers. In one patient, a heavy smoker with markedly elevated blood carbon monoxide levels, an abnormal leftward shift of the OHD curve was observed. This was corrected after discontinuation of smoking. In utilizing these methods, we could not detect consistent abnormalities of $\mathrm{Hb}$ affinity for oxygen at rest in the patients studied, which suggests that a defect in oxygen transport at rest is an unlikely explanation for the symptoms of chest pain in patients with the anginal syndrome and normal coronary arteriograms.
\end{abstract}

\section{INTRODUCTION}

A syndrome of chest pain suggestive of ischemic heart disease but associated with normal coronary arteriog-

This work was presented in part at the 20th Annual Scientific Sessions of the American College of Cardiology, Washington, D. C., February 4, 1971.

Received for publication 11 January 1974 and in revised form 15 April 1974. raphy has become well recognized in recent years (1-9). Individuals with this syndrome comprise approximately $10-20 \%$ of several large series of patients who have undergone angiographic study for suspected coronary artery disease. To further complicate the clinical picture, a number of these patients exhibit objective evidence for myocardial ischemia as manifested by abnormal S-T segment changes after exercise and myocardial lactate production during cardiac catheterization $(4,9)$. Several possible hypotheses have been proposed to explain the underlying symptomatology of this syndrome. These include: disease of the small vessels of the myocardium (10), spasm of the large coronary vessels, maldistribution of coronary blood flow, and abnormalities of oxyhemoglobin dissociation (11). In a recent report, Eliot and Bratt demonstrated significant rightward shifts of oxyhemoglobin dissociation curves at rest in 14 of 15 women with findings suggestive of ischemic heart disease but with normal coronary arteriograms. They further suggested that these abnormalities may play a possible causative role in this condition (11). The purpose of this study, therefore, was to examine the hypothesis of a defect in oxygen transport in similar patients who came to study in our laboratory.

\section{METHODS}

20 patients, 10 men (ages $30-44$ ) and 10 women (ages 37 59) with chest pain suggestive of coronary arteriosclerosis (from 6/1970 to 3/1971) form the basis of this report. Each patient had a history of recurrent chest discomfort established for at least 6 months and often for several years. Several of the patients in the group had been hospitalized on multiple occasions for chest pain suggesting acute coronary insufficiency and were considered to have symptomatic coronary artery disease before the time of study. In each, a thorough search was made for extracardiac sources of chest pain by appropriate clinical studies (including upper gastrointestinal series, cholecystogram, and roentgen examination of the cervicodorsal spine). Individuals with other forms of clinically recognized cardiac disease were excluded from 
this study, as were patients with significant anemia, erythrocytosis, thyroid disease, and chronic renal or pulmonary disease. Antianginal agents (propranolol) were discontinued several (2-3) days before catheterization.

A double two-step exercise test was performed as described previously (12). An exercise test was considered abnormal if $0.5 \mathrm{~mm}$ or greater flat RS-T segment depression occurred during the immediate postexercise period in $50 \%$ of the recorded complexes in any lead.

All subjects underwent diagnostic right and left heart catheterization, selective coronary cinearteriography, and left ventriculography. Pressure measurements were made with Statham P23Db strain gauges (Statham Instruments Inc., Oxnard, Calif.) and recorded on a Sanborn 560 Polybeam photographic recorder (Hewlett-Packard Co., Palo Alto, Calif.). Cardiac output was determined by the indocyanine green dye-dilution technique. Paired blood samples for determination of lactate concentrations were obtained from an indwelling arterial catheter and a second catheter, fluoroscopically positioned within the coronary sinus. Measurements were made at rest and during a stress state with either an isoproterenol infusion or tachycardia induced by atrial pacing. Lactate concentrations were determined in duplicate by using a modification of the method of Horn and Bruns previously reported from this laboratory (13).

Coronary arteriography was performed using dual-field (9-6-inch) (General Electric Medical Systems Div., General Electric Co., Milwaukee, Wis.) image intensification and recorded on 16-mm Ilford Pan F film (Ilford Ltd., Ilford, Essex, England) exposed at 60 frames per s. Multiple projections, including right and left anterior oblique views of each coronary artery, were obtained at both magnifications. In each instance, the films were of good radiographic quality and demonstrated no evidence for intraluminal coronary artery disease. At least three of the authors reviewed each film independently and agreed to the normality of the coronary circulation. Cineventriculograms were performed and analyzed as previously described (14).

Heparinized samples of venous whole blood (WB) ${ }^{1}$ were drawn usually 1 or 2 days after cardiac catheterization when the patient was at rest, breathing room air, and showing no observable evidence of hyperventilation. Red blood cell (RBC) levels of organic phosphate compounds, 2,3-diphosphoglyceric acid (2,3-DPG) and ATP, were determined by the spectrofluorometric method of Lowry, Passonneau, Hasselberger, and Schulz (15). Blood levels of carbon monoxide were determined by a gas chromatographic method (16). Serum inorganic phosphorus was determined by a standard biochemical method.

Oxyhemoglobin dissociation curves using whole blood were performed by using the method described by Duvelleroy, Buckles, Rosenkaimer, Tung, and Laver (17). The reproducibility for values of $P_{50}$ when utilizing this method is within $\pm 0.4 \mathrm{~mm} \mathrm{Hg}$. Data obtained from patients in this study were compared to those of seven normal asymptomatic nonsmoking adults who served as controls using identical methods during the same period of time. Although the entire oxyhemoglobin dissociation curve was determined in each instance, only the values for $\mathrm{P}_{50}$ are indicated in the data reported. $\mathrm{P}_{50}$, the partial pressure of oxygen at $50 \%$ hemoglobin saturation at $\mathrm{pH} 7.4$ and $37^{\circ} \mathrm{C}$, reported here as torr equivalent to $\mathrm{mm} \mathrm{Hg}$, is the accepted convention for numerically defining hemoglobin affinity for oxygen and reflects the relative ability of hemoglobin to bind or release

${ }^{1}$ Abbreviations used in this paper: 2,3-DPG, 2,3-diphosphoglyceric acid; RBC, red blood cell; WB, whole blood. oxygen for use by other tissues. $\mathrm{P}_{50}$, therefore, is used here for comparative purposes only. A more meaningful physiologic value is the $\mathrm{P}_{50}$ at arterial $\mathrm{pH}$. However, assuming that values for arterial and venous $\mathrm{pH}$ in the patient group were within normal limits, the correction to $\mathrm{pH} 7.40$ would introduce no additional technical errors when compared to studies in control subjects. ${ }^{2}$

\section{RESULTS}

In addition to normal coronary arteriograms, all patients in this study exhibited normal left ventricular enddiastolic pressures $(\leq 12 \mathrm{~mm} \mathrm{Hg})$ and cardiac indices $\left(\geq 2.5 \mathrm{l} / \mathrm{min} / \mathrm{m}^{2}\right)$, normal left ventricular end-diastolic volumes $\left(90 \pm 10 \mathrm{ml} / \mathrm{m}^{2}\right)$, ejection fractions $(\geq 55 \%)$, and patterns of contraction. Standard double two-step exercise tests were performed in 18 patients and were positive in 5 .

Myocardial lactate metabolism was studied in $11 \mathrm{pa}-$ tients and was found to be abnormal during stress in 2 individuals. One patient, a man aged 33 , with a history of exertional chest pain completely consistent with classical angina pectoris, demonstrated normal myocardial lactate extraction at rest but lactate production during tachycardia induced by atrial pacing. The other patient, a woman aged 49, exhibited both a positive twostep exercise test and myocardial lactate production during isoproterenol stress. Studies in this patient are described in detail below.

Another patient of special interest included in this group is a woman, aged 52, who presented with the variant form of angina pectoris attributed to Prinzmetal $(19,20)$.

Studies of oxyhemoglobin dissociation in normal control subjects. The data in control subjects is of relevance in interpreting the findings observed in the patient group. Fig. 1 (first panel of each graph) shows values for $P_{50}$ and $R B C$ levels of 2,3-DPG in a normal nonsmoking adult subject over a 9-mo period of time. Values for $P_{50}$ in this subject ranged widely from 25.6 to 29.5 with a mean of $27.6 \pm 1.0$ torr, and $\mathrm{RBC}$ levels of $2,3-\mathrm{DPG}$ from 0.66 to 0.93 with a mean of $0.83 \pm$ $0.08 \mathrm{M} / \mathrm{M}$ hemoglobin.

The combined data of seven additional normal nonsmoking adults over a period of 12 mo are presented in the second panel of each graph in Fig. 1. A similar degree of variability for both values is observed with values for $\mathrm{P}_{50}$ ranging from 23.5 to 29.3 torr $(\mathrm{mm} \mathrm{Hg})$ (mean 26.6 \pm 1.4 torr) and corresponding $\mathrm{RBC}$ concentrations of 2,3-DPG ranging from 0.60 to $0.95 \mathrm{M} / \mathrm{M}$ hemoglobin (mean $0.81 \pm 0.09 \mathrm{M} / \mathrm{M}$ hemoglobin).

Studies of oxyhemoglobin dissociation in patients with the anginal syndrome and normal coronary arterio-

\footnotetext{
${ }^{2} \mathrm{pH}$ at the point of $50 \%$ hemoglobin saturation was determined as previously described (17). Correction to $\mathrm{pH}$ 7.40 was obtained by use of the Bohr factor, i.e., $\Delta \log$ $\mathrm{Po}_{2} / \Delta \mathrm{pH}$, as defined by a standard dissociation curve (18).
} 

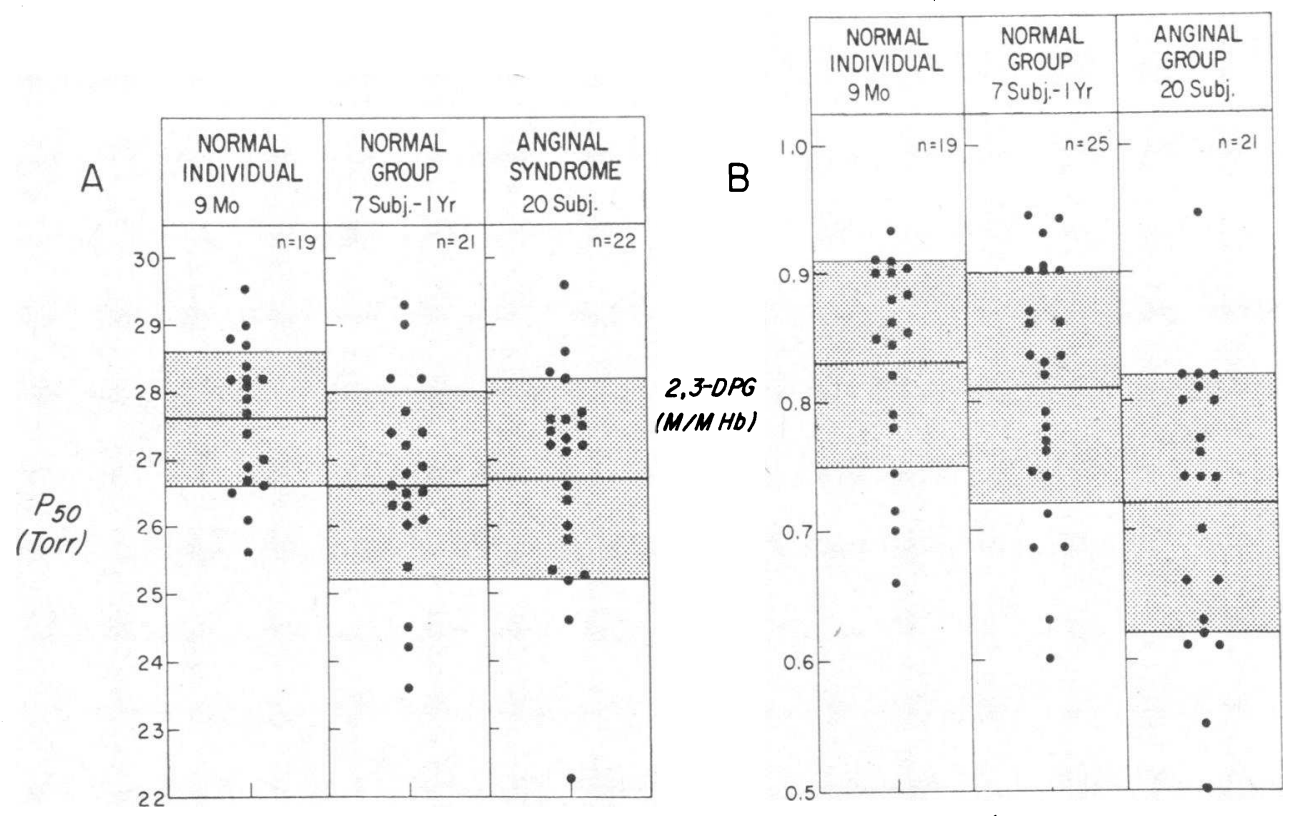

FIGURE 1 Variability of $P_{50}$ and RBC concentrations of 2,3-DPG in a normal nonsmoking individual over a period of 9 mo (first panel of each graph) and in seven other nonsmoking adult subjects over a period of $12 \mathrm{mo}$ (second panel of each graph). $\mathrm{P}_{50}$, the partial pressure of oxygen at $50 \%$ saturation and $\mathrm{pH} 7.4$ is reported as Torricelli (torr), equivalent to $\mathrm{mm}$ $\mathrm{Hg}$, and numerically determines hemoglobin affinity for oxygen. Note the relatively wide range of variation for both values. Mean values $\pm 1 \mathrm{SD}$ are indicated by the shaded areas. In

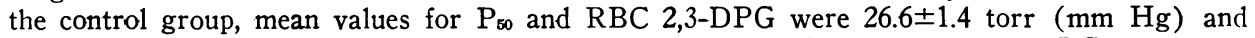
$0.81 \pm 0.09 \mathrm{M} / \mathrm{M}$ hemoglobin $(\mathrm{Hb})$, respectively. Determinations of $\mathrm{P}_{50}$ and $\mathrm{RBC}$ concentrations of 2,3-DPG in patients with the anginal syndrome and normal coronary arteriograms (third panel of each graph) are juxtaposed for comparison. Mean levels for $\mathrm{P}_{50}$ and $\mathrm{RBC}$ 2,3-DPG were $26.7 \pm 1.5$ tor. $(\mathrm{mm} \mathrm{Hg})$ and $0.72 \pm 0.10 \mathrm{M} / \mathrm{M}$ hemoglobin, respectively. No significant statistical differences were observed between the patient and control groups. The two lowest data points in each panel of the patient group represent values for $P_{50}$ and RBC 2,3-DPG, respectively, in the same patient.

grams. Fig. 2 illustrates a typical oxyhemoglobin dissociation curve from a representative patient (C. G.), in this instance, a 49-yr-old female with a long history of recurrent episodic chest pain and a positive twostep exercise test. The values for $P_{50}$ of 27.6 torr $(\mathrm{mm}$ $\mathrm{Hg}$ ) and $\mathrm{RBC} 2,3-\mathrm{DPG}$ of $0.77 \mathrm{M} / \mathrm{M}$ hemoglobin are within the normal range.

The specific values for $P_{50}$ and $R B C$ levels of 2,3-DPG in the entire patient group are also presented in Fig. 1 (third panel of each graph). The control data are juxtaposed for the purpose of comparison. Of 20 patients with the anginal syndrome, studies in 19 fell within or very near the normal range (mean values for the entire group were $\mathrm{P}_{50}, 26.7 \pm 1.5$ torr, and $\mathrm{RBC} 2,3-\mathrm{DPG}$, $0.72 \pm 0.1 \mathrm{M} / \mathrm{M}$ hemoglobin. These include the studies from four of the five patients with a positive two-step exercise test, the previously described male patient with lactate production during stress and the patient with variant angina pectoris. No statistical differences were observed between the two groups.
10 smokers were included in the patient group. Of these, three patients had elevated WB levels of carbon monoxide. Two patients with carbon monoxide concentrations of 0.72 and $0.85 \mathrm{ml} / 100 \mathrm{ml} \mathrm{WB}$ showed minor leftward shifts of the lower portion of their dissociation curves, but values for $\mathrm{P}_{50}$ remained within the normal range. Mean levels of carbon monoxide were normal at $0.14 \pm 0.01 \mathrm{ml} / 100 \mathrm{ml} \mathrm{WB}$ in 10 patients who were nonsmokers.

The lowest data points in each panel of the patient group represent values for $\mathrm{P}_{50}$ and $\mathrm{RBC}$ 2,3-DPG, respectively, in the same patient (M. P.), a 49-yr-old female who was a heavy smoker with a 5-yr history of exertional chest pain and both a positive two-step exercise test and myocardial lactate production (Fig. 3). An initial study performed during the time when she maintained her usual pattern of heavy cigarette smoking (no. 1) demonstrated high blood levels of carbon monoxide at $1.22 \mathrm{ml} / 100 \mathrm{ml} \mathrm{WB}($ or $7 \%$ ) and a significant leftward shift of the dissociation curve with a 


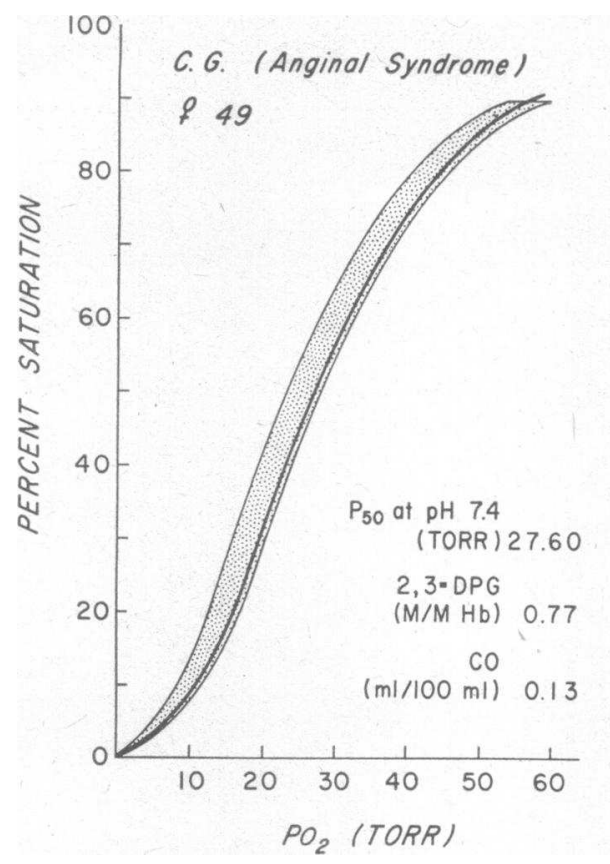

FIGURe 2 Oxyhemoglobin dissociation studies in a representative patient with the anginal syndrome and normal coronary arteriograms. The dissociation curve is superimposed on the normal range (shaded area). Values for $P_{s 0}$ and 2,3-DPG are within normal limits. $\mathrm{Hb}$ is hemoglobin.

$\mathrm{P}_{\mathrm{50}}$ value of 22.4 torr $(\mathrm{mm} \mathrm{Hg})$. A repeat study when she discontinued smoking for 5 days (no. 2) showed a marked fall in blood carbon monoxide, a rightward shift of the dissociation curve to the normal range, but a concomitant decrease in RBC 2,3-DPG. Levels of serum inorganic phosphorus were low in both instances.

\section{DISCUSSION}

The results of oxyhemoglobin dissociation studies in 8 control subjects and 20 patients with the anginal syndrome and normal coronary arteriograms have been presented. In utilizing the methods described, we were unable to detect any consistent abnormality of hemoglobin affinity for oxygen at rest in our patients.

Equal numbers of men and women with this syndrome comprised the study population, in contrast to previous reports in the literature in which females predominated $(3,7)$. Objective laboratory evidence for the presence of myocardial ischemia, as manifested by abnormal RS-T segment depression after exercise or myocardial lactate production, was present in six patients, five of whom were women. The increased frequency of these abnormalities in women as compared to men is consistent with our experience in a large group of such patients with the anginal syndrome and normal coronary arteriograms (9).
Hemoglobin affinity for oxygen. Since nearly all of the oxygen in the blood is carried within the erythrocytes in combination with hemoglobin, the delivery of oxygen to cardiac muscle and other tissues is in part determined by the affinity with which hemoglobin binds or releases oxygen for use by other cells (21). The dynamic character of this affinity relationship between hemoglobin and oxygen over a range of partial pressures for oxygen is graphically represented by the oxyhemoglobin dissociation curve. $\mathrm{P}_{\mathrm{so}}$, the partial pressure of oxygen at $50 \%$ saturation at $\mathrm{pH} 7.4$ and at $37^{\circ} \mathrm{C} \mathrm{nu}-$ merically determines the affinity of hemoglobin for oxygen. Increased hemoglobin affinity for oxygen is characterized by a shift of the oxyhemoglobin dissociation curve to the left of normal, with a lower value than normal for $P_{50}$, while decreased affinity is indicated by a shift of the curve to the right of normal with a higher value than normal for Pso. Recent biochemical studies (22-25) of the hemoglobin-oxygen interaction have established the important role of certain intra-erythrocytic organic phosphate compounds derived from the glycolytic cycle, primarily 2,3-diphosphoglyceric acid (2,3-DPG), in regulating the binding or release of oxygen from the hemoglobin molecule.

One possible point of criticism may be raised regarding the specific values for $P_{50}$ reported in our patients,

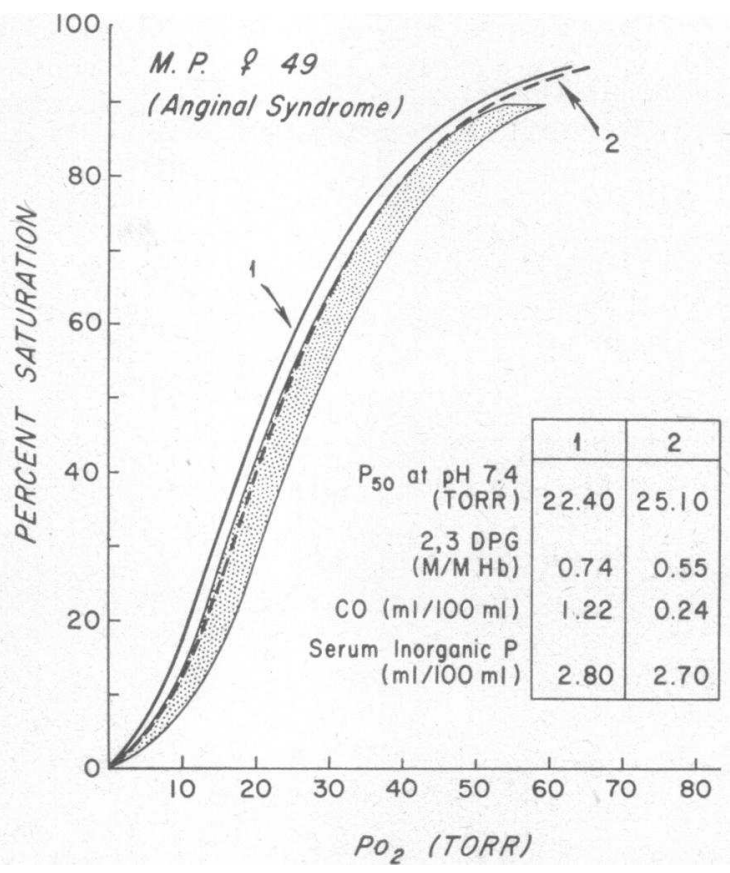

FIGURE 3 Oxyhemoglobin dissociation studies in an unusual patient with the anginal syndrome (see text). The initially observed significant leftward shift of the dissociation curve was associated with high blood levels of carbon monoxide (no. 1). This was corrected after discontinuation of smoking (no. 2). $\mathrm{Hb}$ is hemoglobin. 
i.e., that the correction to $\mathrm{pH} 7.40$ may not be physiologically relevant for the patient whose arterial $\mathrm{pH}$ or the $\mathrm{pH}$ in the coronary arterial bed is markedly different from normal. Based on the usual Bohr factor used for correction (i.e., $\Delta \log \mathrm{Po}_{2} / \Delta \mathrm{pH}=-0.48$ ) we find that an error as high as $0.1 \mathrm{pH}$ units will alter $\mathrm{PO}_{2}$ by \pm 2.5 torr, depending upon an assumption of either too high or too low a $\mathrm{pH}$. We have, however, no evidence that such extensive abnormalities existed in our patients.

Changes in oxyhemoglobin dissociation curves: their physiological significance. Relatively wide ranges of variation in the position of the oxhemoglobin dissociation curves, similar to the data in our control subjects, have previously been reported in normal individuals without manifest hemoglobinopathies $(25,26)$ by other investigators using different methods $(18,27,28)$. Furthermore, within this range of variation, we observed a general direct relationship between specific values for $P_{50}$ and corresponding $\mathrm{RBC}$ levels of 2,3-DPG consistent with the studies of Benesch and Benesch $(22,24)$ and Chanutin and Curnish (23). This normal variability, therefore, makes difficult the interpretation of minor changes in $P_{50}$ or 2,3-DPG reported to occur in studies both in patients with the anginal syndrome and normal coronary arteriograms and those with documented coronary artery disease as being of physiologic importance (29) (vide infra).

Presumably a shift in the oxyhemoglobin dissociation curve should have physiologic consequences only in organs with high differences in arteriovenous oxygen content, such as the heart, brain, and skeletal muscle performing active work $(21,29)$. Studies of transmyocardial oxygen tension have demonstrated a decreasing level of $\mathrm{PO}_{2}$ from the epicardium to the endocardium (30). Hence, one would suspect that such shifts at rest in oxyhemoglobin dissociation curves, particularly when substantiated by appropriate changes in RBC levels of 2,3-DPG, would only be of physiological significance if they altered myocardial oxygen arteriovenous difference, thereby, reflecting a change in myocardial blood flow. This would, at least in theory, be manifested by appropriate changes in coronary venous oxygen content, the fraction of oxygen extracted and resting coronary blood flow (since arterial saturation tends to remain constant at normal levels of $\mathrm{P}_{2}$ ). Such findings have not been described in either patients with the anginal syndrome and normal coronary arteriograms, nor in those with documented coronary artey disease $(4,8,9,31)$.

In one patient (M. P.) with an abnormal leftward displacement of the dissociation curve, this was completely consistent with the high levels of carboxyhemoglobin present. Similar abnormalities have been reported in heavy smokers by several investigators $(32,33)$. While discontinuation of smoking corrected this abnormality, the patient's symptoms of chest discomfort did not abate during this brief period of time. The paradoxical fall in $\mathrm{RBC}$ levels of 2,3-DPG after the rightward shift of the oxyhemoglobin dissociation curve to the normal range and the relatively low levels of serum inorganic phosphorus remain unexplained.

Comments on studies by other investigators. The previous findings reported by Eliot and Bratt (11), although not confirmed by our data, require further comment. The oxyhemoglobin dissociation curves reported in their study were determined by different methods from our own (using $\mathrm{RBC}$ hemolysates and standard tonometric techniques). The rightward shifts in oxyhemoglobin dissociation curves $(>3.1 \mathrm{~mm} \mathrm{Hg}$ than normal) observed in their patients, however, indicate reduced hemoglobin affinity for oxygen and more likely represent a result rather than a cause of myocardial hypoxia (34). More recent studies by these investigators $(35,36)$ utilizing an elaborate stop-flow technique to determine the rate of oxygen release from purified hemoglobin preparations of these patients have demonstrated decreased rates of release of oxygen, a finding not completely consistent with the oxygen-hemoglobin equilibrium (oxyhemoglobin dissociation curves) data reported earlier.

Since the patients described in this report were not studied under conditions of stress, we could not specifically exclude the possibility of an acute defect in oxygen transport during the time of cardiac stress when objective findings of myocardial ischemia were observed. Two recent studies by other investigators, however, are important in this regard. Under conditions of angina pectoris induced by atrial pacing, Schapell et al. (37) demonstrated minor $(0.6-2.9 \mathrm{~mm} \mathrm{Hg})$ increases from control in $\mathrm{P}_{\mathrm{so}}$ of coronary sinus blood in five patients with coronary artery disease. These changes occurred without concomitant changes in RBC levels of 2,3-DPG. In one patient with normal coronary arteriograms, however, no change in $\mathrm{P}_{50}$ was observed during pacing tachycardia, despite the development of ischemic S-T segment depression and myocardial lactate production without concomitant chest pain. In contrast, another study demonstrated minor increases in $\mathrm{P}_{50}$ from control values (rightward shift of the oxyhemoglobin dissociation curve) after pacing tachycardia in 9 of 27 patients with the anginal syndrome and normal coronary arteriograms and in 24 of 76 patients with documented coronary artery disease (38). These findings, particularly in patients with the anginal syndrome and normal coronary arteriograms, are probably of little or no physiologic importance, since normal regulatory mechanisms can augment coronary blood flow many fold to compensate for possible myocardial hypoxia (39). These changes may be 
of importance, however, in patients with coronary arterial lesions during stress where severe coronary flow limitation occurs. In these instances, acute decreases in hemoglobin affinity for oxygen may permit a minor, but perhaps critical, increase in oxygen delivery to ischemic myocardium (40).

\section{ACKNOWLEDGMENTS}

The authors are grateful for the skilled technical assistance of Mr. Peter Higbee and Ms. Charlotte Tung and to Ms. Betty Gottfried and Ms. June Richardson for typing the manuscript.

This study was supported by USPHS Grants PO1-HL11306, ITI-HL-5679, Women's Aid for Heart Research, and Heart Research Foundation, Inc., Boston, Mass.

\section{REFERENCES}

1. Ross, R. S., and G. C. Friesinger. 1966. Coronary arteriography. Am. Heart J. 72: 437-441.

2. Proudfit, W. L., E. K. Shirey, and F. M. Sones, Jr. 1966. Selective cine coronary arteriography, correlation with clinical findings in 1000 patients. Circulation. 33: 901-910.

3. Likoff, W., B. L. Segal, and H. Kasparian. 1967. Paradox of normal selective coronary arteriograms in patients considered to have unmistakable coronary heart disease. N. Engl. J. Med. 276: 1063-1066.

4. Kemp, H. G., W. C. Elliott, and R. Gorlin. 1967. The anginal syndrome with normal coronary arteriography. Trans. Assoc. Am. Physicians Phila. 80: 59-70.

5. Neill, W. A., D. G. Kassebaum, and M. P. Judkins. 1968. Myocardial hypoxia as the basis for angina pectoris in a patient with normal coronary arteriograms. N. Engl. J. Med. 279: 789-792.

6. Dwyer, E. M., Jr., L. Wiener, and J. W. Cox. 1969. Angina pectoris in patients with normal and abnorma coronary arteriograms. Am. J. Cardiol. 23: 639-646.

7. Waxler, E. B., D. Kimbiris, and L. S. Dreifus. 1971. The fate of women with normal coronary arteriograms and chest pain resembling angina pectoris. $\mathrm{Am}$. J. Cardiol. 28: 25-32.

8. Neill, W. A., M. P. Judkins, D. S. Dhindsa, J. Metcalfe, D. G. Kassebaum, and F. E. Kloster. 1972. Clinically suspect ischemic heart disease not corroborated by demonstrable coronary artery disease. Am. J. Cardiol. 29: 171-179.

9. Kemp, H. G., Jr., P. S. Vokonas, P. F. Cohn, and R. Gorlin. 1973. The anginal syndrome associated with normal coronary arteriograms-report of a six year experience. Am. J. Med. 54: 735-742.

10. James, T. N. 1967. Pathology of small coronary arteries. Am. J. Cardiol. 20 : 679-691.

11. Eliot, R. S., and G. Bratt. 1969. The paradox of myocardial ischemia and necrosis in young women with normal coronary arteriograms. Relation to abnormal hemoglobin-oxygen dissociation. Am. J. Cardiol. 23: 633-638.

12. Cohn, P. F., P. S. Vokonas, A. S. Most, M. V. Herman, and R. Gorlin. 1972. Diagnostic accuracy of twostep postexercise ECG. Results in 305 subjects studied by coronary arteriography. J. Am. Med. Assoc. 220: 501-506.
13. Krasnow, N., W. A. Neill, J. V. Messer, and R. Gorlin. 1962. Myocardial lactate and pyruvate metabolism. J. Clin. Invest. 41 : 2075-2085.

14. Herman, M. V., R. A. Heinle, M. D. Klein, and R. Gorlin. 1967. Localized disorders in myocardial contraction. Asynergy and its role in congestive heart failure. N. Engl. J. Med. 277: 222-232.

15. Lowry, O. H., J. V. Passonneau, F. X. Hasselberger, and D. W. Schultz. 1964. Effect of ischemia on known substrates and cofactors of the glycolytic pathway in brain. J. Biol. Chem. 239: 18-30.

16. Collison, H. A., F. L. Rodkey, and J. D. O’Neal. 1968. Determination of carbon monoxide in blood by gas chromatography. Clin. Chem. 14: 162-171.

17. Duvelleroy, M. A., R. G. Buckles, S. Rosenkaimer, C. Tung, and M. B. Laver. 1970. An oxyhemoglobin dissociation analyzer. J. Appl. Physiol. 28: 227-233.

18. Severinghaus, J. W. 1966. Blood gas calculator. J. Appl. Physiol. 21: 1108-1116.

19. Prinzmetal, M., R. Kennamer, R. Merliss, T. Wada, and N. Bor. 1959. Angina pectoris. I. A variant form of angina pectoris. Am. J. Med. 27: 375-388.

20. Whiting, R. B., M. D. Klein, J. V. VanderVeer, and B. Lown. 1970. Variant angina pectoris. N. Engl. J. Med. 282: 709-712.

21. Finch, C. A., and C. Lenfant. 1972. Oxygen transport in man. N. Engl. J. Med. 286: 407-415.

22. Benesch, R., and R. E. Benesch. 1967. The effect of organic phosphates from human erythrocytes on the allosteric properties of hemoglobin. Biochem. Biophys. Res. Commun. 26: 162-167.

23. Chanutin, A., and R. R. Curnish. 1967. Effect of organic and inorganic phosphates on the oxygen equilibrium of human erythrocytes. Arch. Biochem. Biophys. $121: 96-102$.

24. Benesch, R. E., and R. Benesch. 1970. The reaction between diphosphoglycerate and hemoglobin. Fed. Proc. 29: 1101-1111.

25. Bunn, H. F., and J. H. Jandl. 1970. Control of hemoglobin function within the red cell. N. Engl. J. Mrd. 282: $1414-1420$.

26. Ranney, H. M. 1970. Clinically important variants of human hemoglobin. N. Engl. J. Med. 282: 144-152.

27. Van Slyke, D. D., and J. M. Neill. 1924. The determination of gases in blood and other solutions by vacuum extraction and manometric measurement. J. Biol. Chem. $61: 523-573$.

28. Edwards, M. J., and R. J. Martin. 1966. Mixing technique for the oxygen-hemoglobin equilibrium and $\mathrm{Bohr}$ effect. J. Appl. Physiol. 21 : 1898-1902.

29. Laver, M. B. 1972. A fable of our time: oxygen transport, or does the emperor have new clothes? Anesthesiology. 36: 105-106.

30. Kirk, E. S. and C. R. Honig. 1964. Nonuniform distribution of blood flow and gradients of oxygen tension within the heart. Am. J. Physiol. 207: 661-668.

31. Messer, J. V., R. J. Wagman, H. J. Levine, W. A Neill, N. Krasnow, and R. Gorlin. 1962. Patterns of myocardial oxygen extraction during rest and exercise. J. Clin. Invest. 41 : 725-742.

32. Astrup, P., P. Hellung-Larsen, K. Kjeldsen, and $\mathrm{K}$. Millemgaard. 1966. Effect of tobacco smoking on the dissociation curve of oxyhemoglobin: investigations in patients with occlusive arterial disease and in normal subject. Scand. J. Clin. Lab. Invest. 18: 450-457.

33. Ayers, S. M., S. Giannelli, Jr., and H. Mueller. 1970. 
Carboxyhemoglobin ( $\mathrm{COHB}$ ) and the access to oxygen: an example of human counter-evolution. Am. J. Cardiol. 26: 624. (Abstr.)

34. Edwards, M. J. 1970. Correspondence. Hemoglobin "abnormality": perhaps result rather than cause of hypoxia. Am. J. Cardiol. 25 : 377-378.

35. Eliot, R. S., J. M. Salhany, and H. Mizukami. 1970. Angina associated with a decreased rate of oxygen rerease from hemoglobin. Clin. Res. 18: 305. (Abstr.)

36. Guy, C. R., J. M. Salhany, and R. S. Eliot. 1971. Disorders of hemoglobin-oxygen release in ischemic heart disease. Am. Heart J. 82: 824-832.

37. Shappell, S. D., J. A. Murray, M. G. Nasser, R. E.
Wills, J. D. Torrance, and C. J. M. Lenfant. 1970. Acute change in hemoglobin affinity for oxygen during angina pectoris. N. Engl. J. Med. 282 : 1219-1224.

38. Colvard, M. C., J. Chow, E. S. Orgain, and I. Longmuir. 1971. The effects of pacing on oxygen hemoglobin carrying capacity in coronary artery disease. Circ. Suppl. $44: 125$.

39. Berne, R. M. 1964. Regulation of coronary blood flow. Physiol. Rev. 44 : 1-24.

40. Kostuk, W. J., K. Suwa, E. F. Bernstein, and B. E. Sobel. 1973. Altered hemoglobin oxygen affinity in patients with acute myocardial infarction. Am. J. Cardiol. 31: 295-299. 